\title{
Positron Porosimetry Studies of Template Removal from As-Synthesized MCM-41 Silica
}

\author{
R. ZALESKI* AND J. WAWRYSZCZUK \\ Institute of Physics, Maria Curie-Skłodowska University \\ pl. Marii Curie-Skłodowskiej 1, 20-031 Lublin, Poland
}

The course of template removal from MCM-41 pores was investigated by positron annihilation lifetime spectroscopy. Two methods of removal were the object of interest: a decarbonisation after standard calcination procedure and a novel procedure - pyrolysis in vacuum. The shapes of positron lifetime spectra of decarbonised sample are determined by positronium quenching caused by presence of carbon deposit on the surface. Positron annihilation lifetime spectroscopy allows also monitoring the evolution of empty space inside pores. During pyrolysis no conductive carbon is produced, so consecutive stages of template material transformation are easy to observe. In the case of pyrolysis the effect of pore emptying is obtained at temperature $500 \mathrm{~K}$, lower comparing to calcination and decarbonisation $(820 \mathrm{~K})$.

PACS numbers: 78.70.Bj, 36.10.Dr, 61.43.Gt, 81.07.-b

\section{Introduction}

Positron annihilation lifetime spectroscopy (PALS) is a standard method used for determination of small free volume sizes in solids. A probe in PALS is ortho-positronium (o-Ps) - bound state of electron and positron with parallel spins. Ortho-positronium is an unstable particle. In vacuum it annihilates with mean lifetime $142 \mathrm{~ns}$, emitting three gamma quanta. This lifetime is shortened if $o$-Ps is trapped inside a potential well (i.e. electron free volume) in a solid, due to pick-off process - the positron bound in o-Ps undergoes fast two gamma annihilation with one of medium electrons with opposite spin orientation. Shortening of mean lifetime depends on size of the potential well. Widely used Tao-Eldrup formula [1] gives the relation between positronium lifetime and spherical free volume radius if only ground state of particle (o-Ps) in the potential well is occupied. This assumption limits its applicability to free volumes with radius $R<1 \mathrm{~nm}$

*corresponding author; e-mail: radek.zaleski@umcs.lublin.pl 
at the room temperature. Extension of the model to larger voids [2] allows applying PALS as a porosimetry tool in the range of radii from about $0.2 \mathrm{~nm}$ to $100 \mathrm{~nm}$ (micropores and mesopores). Positron porosimetry can compete in this field with other methods used for the investigation of porous material properties, e.g. nitrogen adsorption/desorption method (NA/D). The most important PALS advantage over NA/D is its sensitivity to both closed and opened pores and the ability to make the measurements at various conditions (temperature, pressure etc.). Positron annihilation techniques are used to study the properties of porous materials for a few years, but one encounters new problems appearing when a wide range of lifetimes is measured and analysed. In order to determine capabilities and limits of PALS method, last phase of preparation of ordered porous silica MCM-41 $[3]$ is investigated.

\section{Experimental and materials}

Fast-slow PALS spectrometer with the detectors equipped in $\mathrm{BaF}_{2}$ scintillators was used. Stop energy window was widely opened to acquire as many three gamma annihilation events (continuous energy spectrum) as possible in order to estimate properly the long-lived components intensities. It resulted in time resolution deterioration $\mathrm{FWHM} \approx 300 \mathrm{ps}$, but the main range of interest are $o$-Ps lifetimes (longer than $1 \mathrm{~ns}$ ) which do not depend a lot on possible inaccuracy of determining $p$-Ps (140-180 ps) and free positron (350-500 ps) lifetimes [4]. Additionally, in order to avoid spectrum distortion at high count rate $\left(\approx 10^{3} / \mathrm{s}\right)$, electronic devices rejecting pulse pile-up events were used. Samples were made in sandwich-like configuration enclosing ${ }^{22} \mathrm{Na}$ positron source encapsulated in $8 \mu \mathrm{m}$ thick kapton foil. Vacuum of order of $10^{-3} \mathrm{~Pa}$ kept in the sample chamber prevented $o$-Ps to $p$-Ps conversion in oxygen presence and improves heat isolation. Temperature was controlled by a heater placed below the top of copper rod that could be immersed in liquid nitrogen. Temperature stabilisation was provided by FP-21 (Shimaden) controller. Lifetime spectra were processed using Bayesian methods implemented in MELT subroutine [5]. The carbon content in the sample was estimated using Perkin-Elmer CHN 2400. Pore size distribution was calculated using the BJH procedure from NA/D isotherms measured by Micromeritics ASAP 2010.

Organic template was used to create honeycomb-like structure of parallel cylindrical pores in MCM-41 [6]. Surfactants: $n$-hexadecylpyridinium chloride (sample A) or octadecyl-trimethylammonium bromide (sample B) were used as a template, tetraethyl orthosilicate (TEOS) as a silica source and ammonia as a catalyst. After synthesis, the pores of as-synthetised MCM-41 silica are filled with the surfactant in a form of micelles. Standard procedure of template removal was applied for sample A. The material was calcined in air at temperature rising up slowly (1 degree per minute) to $820 \mathrm{~K}$. After calcination the remnants of the surfactant remain in pores and on a surface of the sample. Mostly it is carbon 
deposit (soot). In order to obtain clear surface, sample A was decarbonised by heating at $820 \mathrm{~K}$ in oxygen flow. In about 3 hour intervals part of the material was taken out from the oven for further investigation by PALS. Sample B was just dried.

\section{Results and discussion \\ 3.1. Decarbonising}

Results of MELT analysis performed on sample A after various time of decarbonising (Fig. 1a) exhibit essential differences. Only short $(\tau<11 \mathrm{~ns})$ lifetimes are observed in the sample just after calcination. The component of lifetime $2.5 \mathrm{~ns}$ predominates in this spectrum. The lifetimes rise after a few hours of heating in oxygen. Four components are found during the first 12 hours of decarbonising. The components can be ascribed to o-Ps annihilation: in intergranular spaces the longest component (LC), inside more or less empty pores - moderate component (MC) and in remnants of surfactant - two short components (SC). After 16 hours the LC dominates the spectra. There is also $1-2 \%$ intense MC ascribed to
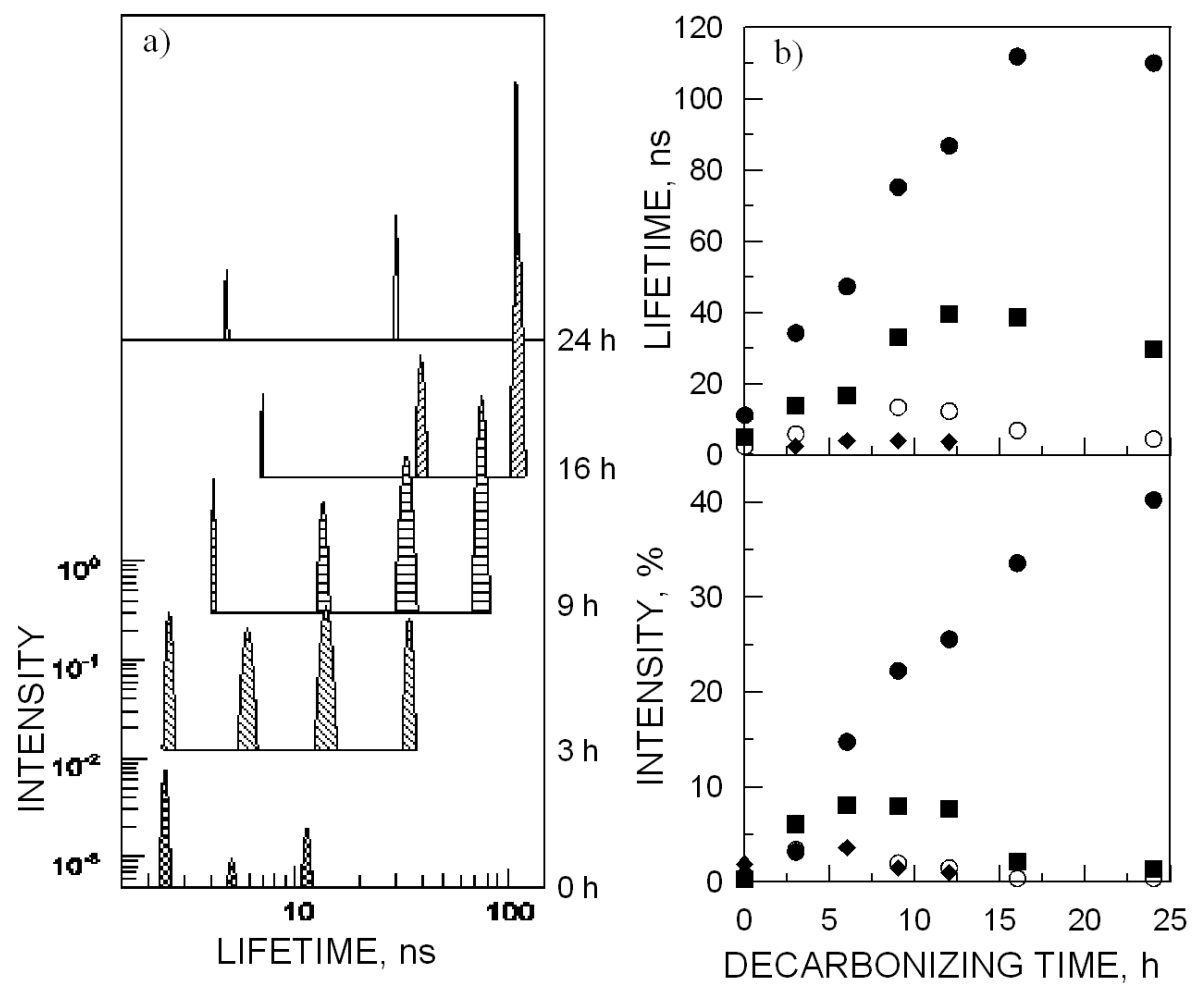

Fig. 1. (a) Histogram of $o$-Ps lifetimes obtained by MELT for calcined MCM-41 samples decarbonised for various times. (b) Lifetimes at maximum of their distribution and intensities of four $o$-Ps components $(\diamond, \circ, \square, \bullet)$ as a function of decarbonising time in calcined MCM-41. 
annihilation in pores. Only one short component is found, which has very low intensity $\left(I_{\mathrm{SC}}<0.5 \%\right)$ so its lifetime cannot be determined accurately. No distinct change is visible in the spectra after further decarbonisation. In order to trace transformation of the sample during decarbonisation, the lifetimes at maximum of distribution and corresponding intensities of the $o$-Ps components are considered (Fig. 1b). Both lifetime and intensity of the intergranular LC rise linearly with decarbonisation time during the first 16 hours. It can be ascribed to diminishing role of $o$-Ps quenching by conducting carbon deposit (soot) on silica surface [7]. Assumption of linear dependence of quenching rate on carbon content leads to an equation describing the change of the LC lifetime

$$
\tau_{\mathrm{LC}}=\left(\tau_{\mathrm{LC}(0 \% \mathrm{C})}^{-1}+C \lambda_{\mathrm{g}(1 \% \mathrm{C})}\right)^{-1}
$$

where $\tau_{\mathrm{LC}(0 \% \mathrm{C})}$ is the $o$-Ps lifetime for no carbon in the sample and $\lambda_{\mathrm{g}(0 \% \mathrm{C})}$ is the rate of quenching per $1 \%$ of carbon. Such approximation is consistent with experimental data for $\tau_{\mathrm{LC}(0 \% \mathrm{C})}=133 \mathrm{~ns}, \lambda_{\mathrm{g}(0 \% \mathrm{C})}=13.6 \mu \mathrm{s}^{-1}$ (Fig. 2a). The rise of the LC intensity reflects increasing probability of Ps formation on increasing carbon free surface of MCM-41 grains.
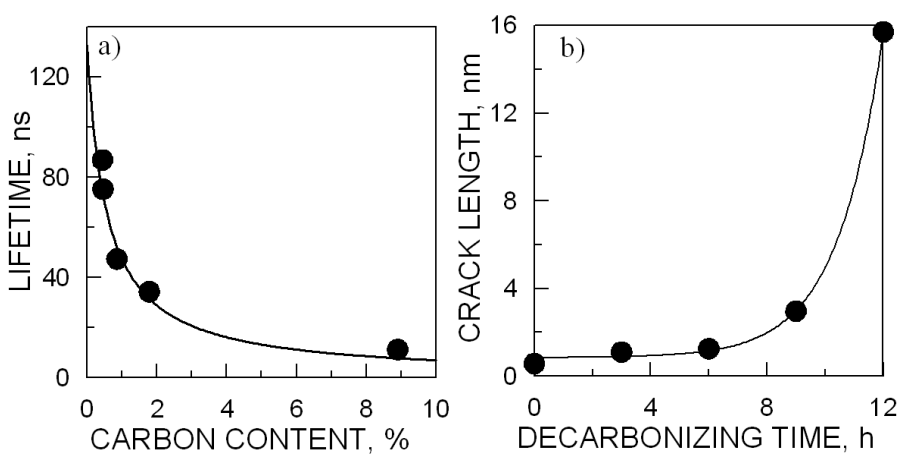

Fig. 2. (a) The longest lifetime at maximum of its distribution versus carbon content in calcined MCM-41 sample decarbonised for various times. (b) Mean length of cracks in the template estimated from o-Ps lifetime using the extended Tao-Eldrup (ETE) model.

Also the change of pore related MC lifetime can be explained by above mechanism, but in this case the MC intensity remains almost constant for 12 hours to finally decrease over four times when decarbonisation is completed. It suggests that origin of the MC lifetime change is different than in the case of the LC. Probably the pores of MCM- 41 are closed by not completely decomposed fragments of surfactant. When this material burns out on the ends of pores, gaps appear in the organic material closing the pores. The shape of cracks is roughly cylindrical. Their radius is constant and equal to pore radius $(R=1.22 \mathrm{~nm})$ known from NA/D isotherms. The rise of the MC lifetime is caused only by the change of crack length. 
In order to observe the evolution of mean crack length with decarbonisation time, the extended Tao-Eldrup model can be used (Fig. 2b). The scenario described above is confirmed by decrease in the SC intensities with decarbonisation time meaning disappearance of template remnants. After 16 hours, when surfactant remnants are removed almost completely, the MC intensity decreases rapidly and the MC lifetime becomes a little shorter than its maximal value after 12 hours of decarbonisation. This is an effect of positronium escape from relatively short (tens of nanometres) open pores to intergranular spaces. If the return to the pores can be neglected, shortened lifetime is described by two-state model [8]:

$$
\tau_{\mathrm{MC}}=\left(\tau_{\text {pore }}^{-1}+\lambda_{\text {escape }}\right)^{-1},
$$

where $\tau_{\text {pore }}=40.73 \mathrm{~ns}$ is calculated using the extended Tao-Eldrup model for infinite cylinder of radius $R=1.22 \mathrm{~nm}$ and $\lambda_{\text {escape }}$ is the probability of positronium escape from the pores. Values of $\lambda_{\text {escape }}$ obtained from Eq. (2) are $1.23 \mu \mathrm{s}^{-1}$ for the spectrum after 16 hours of decarbonising and $9.13 \mu \mathrm{s}^{-1}$ for 24 hours of decarbonising. These results are consistent with decrease in the MC intensity and increase in the LC intensity in these two spectra. Small decrease of the LC lifetime indicates that neglecting the return to the pores is a rough approximation. The difference between spectra after 16 hours and 24 hours of decarbonising is probably not a result of free carbon oxidation, but it is caused by removal of organic material blocking pore ends.

\subsection{Pyrolysis in vacuum}

Another way of the template removal from as-synthesized MCM-41 (sample B) is the pyrolysis in vacuum [9]. Structural changes occurring in the sample during this procedure can be monitored in situ by PALS. The results of the analysis done by MELT for the spectra measured at selected temperatures are shown in Fig. 3a. There are two template related components (SC1 and SC2) with the lifetimes 2-4 ns and an intergranular component (LC) with the lifetime over $100 \mathrm{~ns}$ in as-synthesized MCM-41. The component ascribed to the annihilation in empty pores (MC) is of very low intensity. This component, with the lifetime about $25 \mathrm{~ns}$, becomes well visible at $433 \mathrm{~K}$. At this temperature additional pore related component $\left(\mathrm{MC}^{\prime}\right)$ is found. Its lifetime is about $10 \mathrm{~ns}$. With further temperature rise $\mathrm{MC}^{\prime}$ disappears and the longer lifetime connected with template ( $\left.\mathrm{SC} 2\right)$ changes from 3-4 ns to above $6 \mathrm{~ns}$. All these lifetime changes indicate an alteration of micelle structure and appearance of empty spaces inside the pores. Above $500 \mathrm{~K}$, although $\mathrm{SC} 1$ and $\mathrm{SC} 2$ are still visible, their intensities are very low. The lifetime of $\mathrm{SC} 2$ is about $10 \mathrm{~ns}$. The lifetime of pore related MC component stabilises at almost $40 \mathrm{~ns}$, while lifetime of intergranular LC decreases to the values smaller than $100 \mathrm{~ns}$.

As in Sect. 3.1, the observed changes of component parameters - lifetimes at maximum of distribution and intensities allow to reconstruct the template transformation during its removal from as-synthetised MCM-41. No distinct change of 

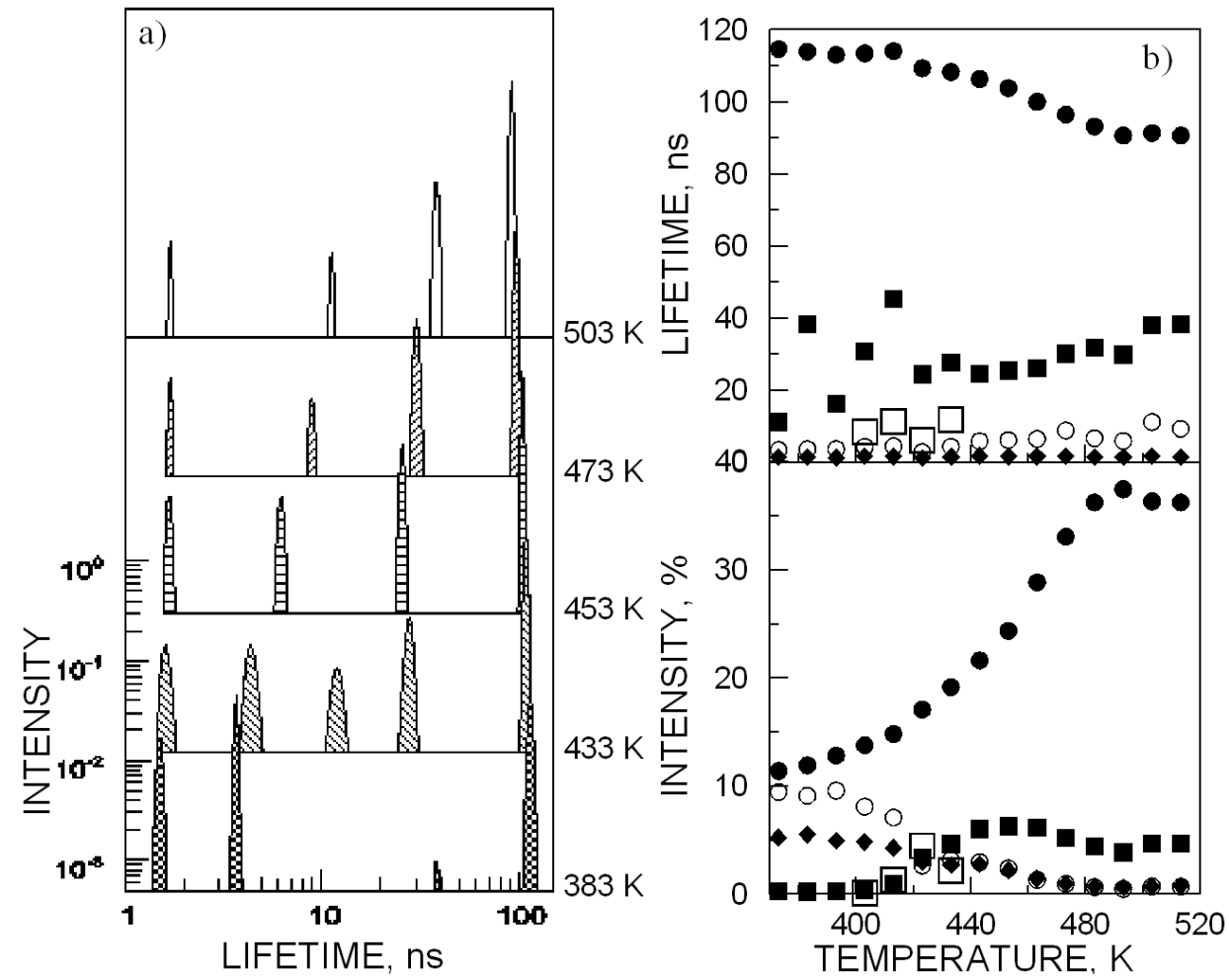

Fig. 3. (a) Histogram of $o$-Ps lifetimes obtained by MELT for as-synthesized MCM-41 samples heated in vacuum at various temperatures. (b) Lifetimes at maximum of their distribution and intensities of five $o$-Ps components $(\diamond, \circ, \square, \mathbf{\square}, \bullet)$ as a function of rising temperature in as-synthetised MCM-41.

the parameters is observed below $400 \mathrm{~K}$. Positronium is trapped mostly in the free volumes in the neighbourhood of micelle and among the grains. Template decomposition begins above $400 \mathrm{~K}$ with appearance of two components of lifetime 10-30 ns ( $\mathrm{MC}$ and $\left.\mathrm{MC}^{\prime}\right)$. Initially, the intensities of these components rise, while intensities of template related components ( $\mathrm{SC} 1$ and $\mathrm{SC} 2$ ) decrease. This indicates positronium escape from free volumes in the micelle to the cracks, which are formed in the template structure. Presence of two crack related components indicates wide distribution of crack lengths. Above $440 \mathrm{~K}$ only one MC component is found. Its intensity stabilises (crack concentration stabilises). On the next stage of template degradation the main reason for lifetime and intensity changes is the loss of the surfactant material due to its thermal decomposition. At temperature below $523 \mathrm{~K}$ decomposition consists mainly in detachment of $\left.\mathrm{N}_{(\mathrm{CH}}\right)_{3}$ head group from the surfactant molecule [10]. This effect is probably correlated to the rise of the SC2 lifetime. It means that longer micellar component is the result of o-Ps annihilation in free volumes placed between the template and the silica wall. Si- 
multaneously, there is no change of the SC1 lifetime, so temperature change does not affect the surrounding of appropriate free volumes. In fact, remaining part of the surfactant is a liquid alkane. Detaching template from the silica walls results of template fragments ability to move and in consequence - change of existing crack sizes. It is confirmed by the rise of the MC lifetime. At the ends of pores, the moving template fragments fall out from the pores. This process causes the rise of the intergranular component LC intensity due to the increase in the total sample surface (empty pores) combined with positronium escape from the pores. Above $470 \mathrm{~K}$ the intensity of crack related component MC decreases indicating final stage of pore emptying. At this temperature the sum of SC1 and SC2 intensities drops to $1.5 \%$. Above $500 \mathrm{~K}$ there are no significant changes in the positron lifetime spectra. Most of the positronium annihilates outside the silica grains, but the fraction of positronium annihilating in empty pores with the lifetime of about $38 \mathrm{~ns}$ is clearly visible. NA/D isotherms confirm that the degree of template removal in vacuum at $500 \mathrm{~K}$ is similar to that after standard calcination and decarbonisation procedure conducted at $820 \mathrm{~K}$ in air.

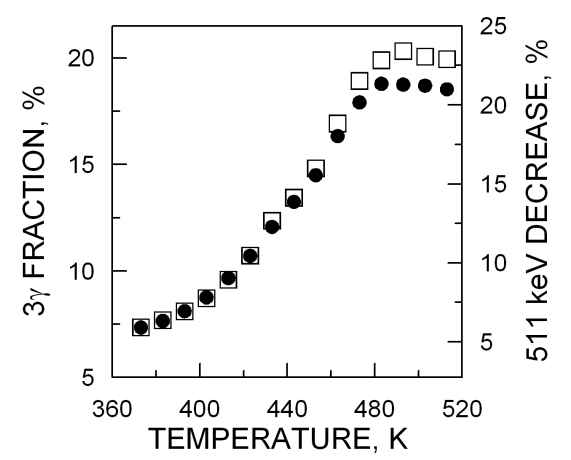

Fig. 4. Fraction of positron $3 \gamma$ annihilation calculated from PALS spectra (circles) and relative decrease in $511 \mathrm{keV}$ peak (open squares).

In PALS spectra the intensities of long-lived components are distorted due to different detection efficiency of $3 \gamma$ and $2 \gamma$ annihilation events. In order to estimate this distortion, fraction of $3 \gamma$ annihilation calculated from PALS spectra [11] is compared to relative decrease in the $511 \mathrm{keV}$ peak intensity in ${ }^{22} \mathrm{Na}$ gamma energy spectrum, which is proportional to increase in $3 \gamma$ annihilation intensity. The changes of both values during pyrolysis in vacuum are shown in Fig. 4. Scales were adjusted to obtain agreement of each method results for initial low $3 \gamma$ fraction, when the distortion is negligible. For higher $3 \gamma$ intensities, at temperatures above $430 \mathrm{~K}$, the fraction of $3 \gamma$ annihilation obtained from PALS lie below values anticipated basing on relative decrease in the $511 \mathrm{keV}$ peak intensity. Thus intensities of the long-lived components in PALS spectra are probably slightly underestimated. 


\section{Conclusions}

At least 16 hours of decarbonisation is needed to obtain MCM-41 silica with empty pores. Until that time external surface of the grains is covered by the conducting carbon (soot) which causes positronium quenching. The pores in MCM-41 silica are filled with larger template remnants separated by gaps where positronium can be formed and trapped. After complete removal of organic material, majority of positronium escapes from quite short pores to intergranular spaces. It causes a decrease in lifetime and intensity of the component related to open pores in comparison to the lifetime and the intensity observed when pores are closed.

In order to remove template by pyrolysis in vacuum lower temperature $(500 \mathrm{~K})$ is needed than in the case of standard calcination procedure $(820 \mathrm{~K})$. Heating without oxygen leaves no free carbon in the sample, so decarbonising is not needed. In vacuum the surfactant is decomposed to volatile trimethyloamine and alkane lasting in pores as a liquid. During further heating alkane is sucked out of pores as a whole. MCM-41 obtained this way is close to that after calcination and decarbonising.

\section{Acknowledgments}

Authors thank T. Goworek for valuable discussions and remarks.

\section{References}

[1] D. Lightbody, J.N. Sherwood, M. Eldrup, Chem. Phys. 93, 475 (1985).

[2] T. Goworek, K. Ciesielski, B. Jasińska, J. Wawryszczuk, Chem. Phys. Lett. 272, 91 (1997).

[3] C.T. Kresge, M.E. Leonowicz, W.J. Roth, J.C. Vartuli, J.S. Beck, Nature 359, 710 (1992).

[4] R. Zaleski, Acta. Phys. Pol. A 110, 729 (2006).

[5] A. Shukla, M. Peter, L. Hoffman, Nucl. Instrum. Methods Phys. Res. A 335 , 310 (1993).

[6] M. Grün, K.K. Unger, A. Matsumoto, K. Tsutsumi, in: Characterization of Porous Solids IV, Eds. B. McEnaney, T.J. Mays, J. Rouquerol, F. Rodríguez-Reinoso, K.S.W. Sing, K.K. Unger, The Royal Society of Chemistry, London 1997, p. 81.

[7] J. Wawryszczuk, J. Goworek, R. Zaleski, T. Goworek, Langmuir 19, 2599 (2003).

[8] M. Eldrup, N.J. Pedersen, J.N. Sherwood, Phys. Rev. Lett. 43, 1407 (1979).

[9] R. Zaleski, J. Wawryszczuk, J. Goworek, A. Borówka, T. Goworek, J. Colloid Interface Sci. 262, 466 (2003).

[10] F. Kleitz, W. Schmidt, F. Schüth, Microporous Mesoporous Mater. 44-45, 95 (2001).

[11] B. Jasińska, J. Wawryszczuk, R. Zaleski, Acta Phys. Pol. A 107, 821 (2005). 